\title{
The Inflationary Consequences of a Currency Changeover: Evidence from the Michelin Red Guide *
}

\author{
Fabrizio Adriani $\rangle_{\text {Giancarlo Marini; Pasquale Scaramozzino }}^{\S}$ \\ January 18, 2004
}

\begin{abstract}
This paper examines the inflationary consequences of a currency changeover in a simple model of the catering market. It suggests the hypothesis that the change in cash denomination may act as a coordination device shifting the industry to a high-price equilibrium. Empirical evidence on the changeover to the euro based on data from the Michelin Red Guide strongly supports the predictions of the model against competing explanations. A permanent change in relative prices has taken place in Euroland, with redistributional effects in favour of some segments of the catering sector.
\end{abstract}

JEL-classification: D43, D82, E31, F33. Keywords: Restaurants, Tourists, Euro, Currency Changeover.

${ }^{*}$ We thank G. Corsetti, C. Engel, N. Giammarioli, F. van der Ploeg and, in particular, A. Ciccone for comments and suggestions.

${ }^{\dagger}$ Department of Economics - University of Bristol - 8 Woodland Road Bristol BS8 1TN, UK. E-mail f.adriani@bristol.ac.uk

${ }^{\ddagger}$ Department of Economics - University of Rome "Tor Vergata".

$\S$ Department of Financial and Management Studies - SOAS - University of London. 


\section{Introduction}

The introduction of the Euro notes and coins on 1st January 2002 to replace national currencies has sparked off an intense debate on the economic consequences of the changeover. According to a conventional view, the change of currency should not have had any effects on relative prices, since money is simply a "veil". The main direct consequence of the changeover should have been the reduction in transaction costs associated to a variety of currencies in the European Union. The adoption of a common currency among a large number of European countries generates economies of scale and promotes a greater degree of efficiency in production. The reduction in price uncertainty would increase welfare through the improvements in the allocation of resources. Increased transparency of prices would also enhance competition both across and within countries. Of course, all these benefits would be greater the closer the member countries were to satisfying the requirements for an optimal currency area.

On the other hand, a much feared cost in the public opinion, often reported by the media, was the perception of generalized price inflation triggered by the currency changeover. Price increases in restaurants and in the service sector have indeed taken place following the introduction of the Euro. These increases have mainly been attributed to the dynamics of costs in the food sector due to inclement weather conditions (European Central Bank [March 2002] pp. 31-32; European Central Bank [April 2002], p. 19). It has been argued that "the extent of the cash changeover effect has been relatively limited, and should be temporary" (European Central Bank [July 2002] p. 22).

Although price increases might have been una tantum with no substantial effect on the inflation rate, the change in relative prices may however have been permanent. This paper provides support for the layman's view that the changeover resulted in a permanent change in relative prices, and will have significant and long-lasting allocative and distributional effects. The switch from national currencies to the Euro has acted as a device that has led firms to co-ordinate their expectations on pricing behaviour. The exogenous change in cash denomination has thus shifted the industry to a higher-price equilibrium. In other words, the widespread concerns about possible generalized price increases in these industries associated with the introduction of the new currency have generated self-fulfilling inflationary expectations.

We analyse the issue of the inflationary consequences of a currency changeover by focusing on the catering market. The market for restaurant services is ideally suited to our purposes due to its characteristics of segmentation and imperfect information. We develop a simple model where customers 
have heterogeneous information sets. There are informed agents (Locals) who know the quality of individual restaurants and uninformed agents (Tourists) who do not. Restaurants choose whether to specialize in dealing only with a single type of customer or to attract both types. The equilibrium strategy of a restaurant depends on its quality and on its potential to attract tourists. Low quality restaurants and restaurants located in tourist areas are more tempted to set a price that leaves negative surplus to their customers, exploiting the imperfect information of tourists. This is traded off by the cost of losing potential local customers.

The relationship between expected and current price may exhibit multiple equilibria. Agents will co-ordinate on a given equilibrium according to their expectations about the strategy restaurants will select and the price at which tourists will accept to trade. Also, the model predicts that restaurants adopting different strategies will react with different responses to a shift from a low price equilibrium to a high price equilibrium. Such predictions allow us to test the validity of our explanation by focusing on the interactions between the structure of the market and price expectations.

Our approach enables us to verify our interpretation relative to alternative suggested explanations, such as: (i) increase in food costs due to bad weather, (ii) delayed and overdue adjustments of menus, or (iii) rounding-up of prices in the new currency. By looking at restaurants both inside and outside the Euro area, we can only test the validity of our predictions against (i). However, by also exploiting the heterogeneity in the determinants of restaurants' equilibrium strategies we are able to discriminate between our model and all the competing explanations. The model predicts that price increases will mainly occur among the restaurants that specialize in attracting tourists. This enables us to reject explanations based on (i)-(iii), that would instead apply to all restaurants in the euro area.

Our empirical results are based on data from the Michelin Red Guide which has the merit to provide information about the quality and the potential for attracting tourists of each restaurant. On the other hand, restaurants included by the guide are selected on the basis of the price-quality combination offered to their customers. This suggests that our results might underestimate the real impact of the changeover. Despite this conservative sample selection our results strongly support our expectation-driven view of price inflation following the changeover, against all competing alternatives. Hence, a permanent change in relative prices has occurred with the introduction of the Euro, with clear redistributional consequences.

The plan of the paper is as follows. Section 2 develops a theoretical model of the catering market. Section 3 discusses the inflationary effects of a currency changeover. Section 4 presents our econometric results. Section 
5 concludes.

\section{The Model}

We develop a model of the catering sector based on imperfect information and market segmentation. We assume that restaurants attract two types of customers endowed with different information sets: regular customers who know the quality of a restaurant in advance ("Locals") and all other customers ("Tourists"). Restaurants differ both in the quality of their meals and in the probability to be visited by tourists. Establishments situated in more strategic locations are likely to attract more tourists than local customers. ${ }^{1}$

Quality is an exogenous characteristic of restaurants. These also differ in their capacity to be matched with uninformed customers. Our focus is on how restaurants' choice of whether to rip off or to be "honest" depends on the likelihood to be matched with uninformed consumers and on reservation prices. We show how strategic interactions between restaurants and customers eventually lead to multiple equilibria.

We treat quality as an "experience" characteristic of the good (Nelson [1970]; Cooper and Ross [1984]). This implies that quality can be discovered only after consuming the good. Chan and Leland [1982] consider the problem of "search goods" (i.e. those goods where quality can be observed after bearing some information cost). Real world goods usually fall in between these two categories. Despite tourists being able to obtain some information on the quality of a restaurant, for instance by purchasing a tourist guide, the restaurant example resembles more the case of an experience good (von Ungern-Sternberg and von Weizsacker [1985]). The rationale for the private information of the Locals in our model thus comes from the assumption that they have "experienced" every restaurant at some point in time in the past, whereas tourists have not.

Restaurants and customers are matched through an exogenous Poisson process. The use of stochastic matching captures typical departures of the catering sector from competitive markets such as the presence of monopolistic rents, price setting sellers and barriers related to the free entry of new firms in the presence of equilibrium profits.

\footnotetext{
${ }^{1}$ Models with informed and uninformed consumers have been widely considered in the literature since Salop and Stiglitz [1977]; agents bear different costs in gathering information about prices while quality is not an issue. Cooper and Ross [1984] consider a model with heterogenous information where the entry of firms selling low quality goods at high price (ripoffs) may cause the uninformed consumers to exit the market.
} 
At each point in time, a new cohort of Tourists of size $\gamma$ arrives in town. Tourists remain in town until the match with the first restaurant occurs. We assume that each Tourist departs after the first match. There is also a constant unit mass of infinitely lived Locals.

Both customer-types want to maximise their surplus:

$$
U\left(x_{j}\right)=v\left(x_{j}\right)-p
$$

where $p$ is price and $v\left(x_{j}\right)$ is the utility associated with a meal of quality $x_{j}$, $j \in\{L, M, H\}$. Locals can exploit private information in the sense that they always know in advance the quality of a given restaurant whereas Tourists learn it only after the meal. When a match occurs, the restaurant makes the customer a take it or leave it offer about the price of the meal. Thus, there is no bargaining or, put differently, the restaurant has all the bargaining power. We assume that the restaurant is not able to discriminate between locals and tourists (for instance, because it is required to display a menu before the meal).

When the offer is made, the customer can decide whether to accept it or decline it. Thus, whenever $v\left(x_{j}\right) \geq p$ the local will accept the offer.

The tourist does not know the quality in advance and, therefore, he must rely on expectations. His ex-ante utility is $E\left(v\left(x_{j}\right) \mid I_{T}\right)-p$ if he accepts and 0 if he refuses $\left(I_{T}\right.$ is his information set).

Restaurants are heterogenous in two characteristics: they are different in quality and in the probability to be spotted by tourists. The idea is that while locals can be assumed to spread evenly across all the restaurants in the town, Tourists are likely to find some places more easily than others. For instance, an airport restaurant is likely to attract more tourists than a restaurant in an industrial area. Let $\lambda_{i}$ be the Poisson arrival rate of tourists for restaurant $i$. We assume that $\lambda_{i} \in[\underline{\lambda}, \bar{\lambda}]$ is uniformly distributed. The arrival rate of Locals $\lambda$ is instead assumed to be constant across all the restaurants. In order to have constant population we assume $\gamma=\bar{\lambda}-\underline{\lambda}$. This implies that the population size of Tourists is equal to 1 at any point in time.

Restaurants are also different in quality. There are top quality $x_{H}$, medium quality $x_{M}$ and low quality $x_{L}$ restaurants. Defining $\mathcal{X} \equiv\left\{x_{H}, x_{M}, x_{L}\right\}$, the space of restaurants is given by $\Omega=\mathcal{X} \times[\underline{\lambda}, \bar{\lambda}]$. Producing meals is costly and the unit cost depends on quality: $c\left(x_{L}\right)<c\left(x_{M}\right)<c\left(x_{H}\right)$.

\subsection{Pricing Strategies}

Since the restaurant cannot discriminate, per customer profits in the case of a Local must be the same as in the case of a Tourist. The only difference 
is under which conditions a Local or a Tourist will accept the offer. More precisely, a local will accept it only if the price does not exceed his reservation price $p_{L}^{*}\left(x_{j}\right)=v\left(x_{j}\right)$ whereas a tourist will accept it only if $p \leq E\left(v\left(x_{j}\right) \mid I_{T}\right)$ which implies a reservation price $p_{T}^{*}=E\left(v\left(x_{j}\right) \mid I_{T}\right)$. Given the Poisson nature of the process, a restaurant with per customer profits $\pi$, arrival rate of tourists $\lambda_{i}$ and arrival rate of locals $\lambda$ has a discounted value of profits at time zero equal to:

$$
\begin{array}{r}
\Pi=\int_{0}^{\infty} e^{-\delta \tau} \sum_{s=0}^{\infty} \frac{\left(\lambda_{i} \tau\right)^{s}}{s !} e^{-\lambda_{i} \tau} s \pi d \tau+\int_{0}^{\infty} e^{-\delta \tau} \sum_{s=0}^{\infty} \frac{(\lambda \tau)^{s}}{s !} e^{-\lambda \tau} s \pi d \tau= \\
=\frac{\lambda+\lambda_{i}}{\delta} \pi
\end{array}
$$

where $\delta$ is the discount rate. In order to get both Locals and Tourists a restaurant of quality $x_{j}$ should set a price $p=\min \left[p_{T}^{*}, p_{L}^{*}\left(x_{j}\right)\right]$. Therefore, profits per customer are $\pi=\min \left[v\left(x_{j}\right), p_{T}^{*}\right]-c\left(x_{j}\right)$. On the other hand, a restaurant could find it optimal to set a price that is capable to capture only Locals or only Tourists. Let $s_{P R}, s_{R T}, s_{R R}$ be the strategies of restaurants dealing, respectively, with both Locals and Tourists, only with Tourists and only with Locals. For mnemonic purposes, let us call the types of restaurants associated with three strategies respectively: Popular Restaurants (PR), Restaurants for Tourists (RT) and Restaurants for Regulars (RR). The associated profits are:

$$
\begin{gathered}
\Pi_{P R}=\frac{\lambda+\lambda_{i}}{\delta}\left(\min \left[p_{T}^{*}, v\left(x_{j}\right)\right]-c\left(x_{j}\right)\right) \\
\Pi_{R T}=\frac{\lambda_{i}}{\delta}\left(p_{T}^{*}-c\left(x_{j}\right)\right) ; p_{T}^{*}>v\left(x_{j}\right) \\
\Pi_{R R}=\frac{\lambda}{\delta}\left(v\left(x_{j}\right)-c\left(x_{j}\right)\right) ; p_{T}^{*}<v\left(x_{j}\right)
\end{gathered}
$$

Whenever a restaurant sets a price that is the minimum between the two reservation prices it sells to both groups. When instead it sets the maximum of the pair it only gets one group. Note that RR leave zero surplus to their customers. This is a consequence of the information rent they enjoy and of the assumptions on bargaining power. Since all their customers (Locals) know their quality, and RR know they know, this type of restaurants is able to appropriate all consumers' surplus. 
Let us now compare the profits under the different strategies. In order to assess which is the optimal strategy, restaurants use their expectation on the Tourist's average reservation price $\bar{p}_{T}^{*}$ :

$$
\begin{array}{r}
\Pi_{P R} \geq \Pi_{R T} \Leftrightarrow \lambda_{i} \leq \frac{v\left(x_{j}\right)-c\left(x_{j}\right)}{\bar{p}_{T}^{*}-v\left(x_{j}\right)} \lambda \equiv \lambda_{j}^{R T} ; \quad \text { if } v\left(x_{j}\right)<\bar{p}_{T}^{*} \\
\text { and } c\left(x_{j}\right) \leq \bar{p}_{T}^{*}
\end{array}
$$

for $j=L, H, M$. This says that when $v\left(x_{j}\right)<\bar{p}_{T}^{*}$ there exists a cut-off value $\lambda_{j}^{R T}$ for $\lambda_{i}$ above which the restaurants will choose to neglect the Locals and only work with Tourists. In general, if $v\left(x_{j}\right)-c\left(x_{j}\right)$ is roughly constant or increasing in the quality, then the cut-off will be increasing in the restaurant's quality.

Proposition 1. If profits per costumer $v\left(x_{j}\right)-c\left(x_{j}\right)$ are not sharply decreasing in $x_{j}$ then low quality restaurants are more likely to become restaurants for tourists than other restaurants.

It is useful to note that $p_{T}^{*} \in\left[c\left(x_{L}\right), v\left(x_{H}\right)\right)$ and, therefore, for expectations to be consistent we also require $\bar{p}_{T}^{*} \in\left[c\left(x_{L}\right), v\left(x_{H}\right)\right)$.

We can now show that a Tourist suffers an ex-post loss when he goes to a Restaurant for Tourists.

Lemma 1. A Tourist matched with a RT he experiences a negative surplus.

Proof. Suppose he were not, then Locals would also go to the RT. But then the restaurant would no longer be a RT.

A restaurant could also decide to become a Restaurant for Regulars. In this case it would set a price which is equal to $v\left(x_{j}\right)>\bar{p}_{T}^{*}$ and deal only with Locals thereby extracting all their surplus. It is straightforward to show that if a restaurant prefers the RT strategy to the PR strategy, then it also prefers to be a PR rather than a RR. Consider the choice between being a Popular Restaurant and a RR:

$$
\begin{array}{r}
\Pi_{R R} \geq \Pi_{P R} \Leftrightarrow \lambda_{i} \leq \frac{v\left(x_{j}\right)-\bar{p}_{T}^{*}}{v\left(x_{j}\right)-c\left(x_{j}\right)} \lambda \equiv \lambda_{j}^{P R} ; \quad \text { if } v\left(x_{j}\right)>\bar{p}_{T}^{*} \\
\text { and } c\left(x_{j}\right) \leq v\left(x_{j}\right)
\end{array}
$$

for $j=\{L, M, H\}$. A restaurant $i$ of quality $j$ will become a restaurant for regulars if $\lambda_{i} \leq \lambda_{j}^{P R}$.

Proposition 2. If profits for costumer $v\left(x_{j}\right)-c\left(x_{j}\right)$ are not sharply decreasing in quality $x_{j}$ then top quality restaurants have more incentives to become restaurants for regulars. 
Popular Restaurants must charge a price that is not above the utility associated with their quality in order to attract both categories of customers. Thus, when both tourists and regulars are matched with a popular restaurant, they experience a non-negative surplus.

\section{Currency Changeovers and Sectoral Prices}

In this section we discuss the possibility of multiple equilibria and its consequences on prices. The individual tourist's payoff is affected both by the behaviour of the mass of tourists and by restaurants' strategies. If restaurants expect low reservation prices, the average quality of restaurants which find it optimal to deal with tourists is also low. Thus, Tourists' willingness to pay turns out to be low as well in a self-fulfilling fashion. On the other hand, if expectations are revised upward, the economy could jump to an equilibrium with high prices.

\subsection{Equilibria}

Assume that a Tourist can compute the average reservation price of the other Tourists and let $g\left(\bar{p}_{T}^{*}\right)$ denote the conditional quality function: $g\left(\bar{p}_{T}^{*}\right) \equiv$ $E\left(v\left(x_{j}\right) \mid \bar{p}_{T}^{*}\right)$.

Definition 1. A Symmetric Nash Equilibrium (SNE) is a level of $p_{T}^{*}$ such that $p_{T}^{*}=g\left(\bar{p}_{T}^{*}\right)$ and $p_{T}^{*}=\bar{p}_{T}^{*}$.

In the equilibrium, both the restaurateur and the individual tourist perfectly predict the tourists' average reservation price.

It is clear from the previous section that the expected quality is strongly discontinuous in the average reservation price. The strategy chosen by a restaurant of quality $j$ crucially depends on whether $\bar{p}_{T}^{*} \lesseqgtr v\left(x_{j}\right)$. A taxonomic analysis of the possible equilibria is contained in Appendix. We show there how the function $g\left(\bar{p}_{T}^{*}\right)$ is characterised both by non-linearity and discontinuities which may generate multiple equilibria. The reason is the strategic interaction between the single Tourist, the mass of tourists and restaurants. The mass of Tourists affects the single Tourist's payoff by influencing the pricing strategy of restaurants. When restaurants expect a low reservation price $\bar{p}_{T}^{*}$, the average quality of restaurants willing to deal with Tourists is low. In fact, top quality restaurants have little incentive to charge a price capable to attract uninformed costumers. This, in turn, implies that Tourists, predicting such behaviour, will actually have a low reservation price. On the other hand, when expectations of high reservation price prevail, the 
equilibrium price will be high as well. It is important to note that a shift may occur without any change of aggregate quality in the economy. An example with two equilibria is reported in Fig. 1.

A characteristic feature of the equilibria in this model is:

Proposition 3. A simultaneous and coordinated revision in expectations of the tourists' reservation price may cause the price to jump from an equilibrium to another one.

In the event of a jump from a low price to a high price equilibrium, restaurants will behave differently according to their market strategies. Let $p_{T}^{0}$ and $p_{T}^{1}>p_{T}^{0}$ be two possible equilibrium prices. At some point in time $t_{0}$ the economy is in $p_{T}^{0}$. Assume that at time $t_{1}$ expectations are revised and the average reservation price jumps from $p_{T}^{0}$ to $p_{T}^{1}$. Restaurants for Tourists will always charge the higher price $p_{T}^{1}$. Popular restaurants will decide to charge $p_{T}^{1}$ as well. Other popular restaurants will increase their prices if $p_{T}^{0}$ was lower than the locals' reservation price and will leave prices unchanged otherwise. In addition, some restaurants which were charging $v\left(x_{j}\right)>p_{T}^{0}$ will find it profitable to charge the new price $p_{T}^{1}<v\left(x_{j}\right)$, reducing thus their prices. Other restaurants for regulars will leave prices unchanged. The overall effect on the mass of popular restaurants after the jump is ambiguous. Thus, an important implication of this model is:

Proposition 4. In the presence of a jump from a low to a high price equilibrium, restaurants will behave differently according to their strategies. In particular:

1. RT will increase their prices.

2. some PR will increase their prices, some will leave them unchanged, some will reduce their prices.

3. RR will leave prices unchanged.

We conjecture that the introduction of the Euro may have worked as a coordination mechanism that allowed a switch from a low-price to a highprice equilibrium. The media may have played an important role in affecting expectations. Before the introduction, the main issue was the rounding up of prices converted in Euros from prices expressed in national currencies ${ }^{2}$.

\footnotetext{
${ }^{2} \mathrm{~A}$ brief summary of the events leading to the introduction of the euro. In December 1998 fixed exchange rates between euro and national currencies were announced by the
} 
As soon as the euro replaced the old national currencies, consumers' associations and research institutions started to express concerns that sellers might increase their prices, exploiting consumers' difficulties in the conversion. The media massively covered such stories. This process may have worked as a device to coordinate expectations toward a high price equilibrium. Proposition 4 shows that Restaurants for Tourists are likely to increase their prices when faced with such scenario. On the other hand, it is possible to argue that conversion difficulties are the same for both Locals and Tourists. By contrast, Locals are often regular customers. This implies that they are more likely to remember old prices - and compare them with the new prices after the conversion - than Tourists. Thus, restaurants dealing with Locals must necessarily be more cautious than other restaurants in raising their prices. The model predicts that Locals will accept an increase in price as long as they continue to experience a positive surplus which can be assessed ex ante, since they know the quality. By contrast, tourists have much more difficulties to compare old prices with new ones, even when they have no problems with the conversion calculations. They also cannot rely on accurate knowledge about quality. Both these sources of imperfect information make their choice problematic. In other words, the daily warnings about price increases due to "difficulties in converting" may have triggered a shift to a high price equilibrium through a self-fulfilling mechanism.

We test the empirical implications of the model in the next section.

\section{Evidence}

\subsection{Data Description}

We collect data from the Michelin Guide [2002 and 2003 editions] ("Main Cities of Europe") for six countries: Denmark, France, Germany, Italy, Sweden and UK. All the six countries are long-standing members of the European Union. France, Germany, and Italy have adopted the EURO as a new national currency since 2002. Denmark, Sweden, and UK retained their national currencies. We consider restaurants for which observations are available for both the 2002 and 2003 editions. Despite euro notes having been introduced since January 2002, the 2002-2003 period is the most appropri-

national central banks of the twelve countries joining the euro. Starting from January 1999, the euro became the official currency in these countries, although no notes were issued in euros. Since January 2002 notes in national currency started to be replaced by notes in euros. The replacement process was completed on different dates across the twelve countries. 
ate to capture the effect of the changeover on prices for two reasons. First, the guide is published by the end of March each year and some countries experienced a double currency regime for the first months of 2002. Secondly, during 2002 some local authorities have kept prices under strict monitoring in order to avoid unfair rounding up.

We use the following dummies: EURO (which takes value 1 when the restaurant is in the euro area), RT (which takes value 1 if the restaurant is classified as a Restaurant for Tourists), PR (which takes value 1 if the restaurant is classified as a popular restaurant), $R R$ (which takes value 1 if the restaurant is classified as for regular customers).

As noted in section 2, the determinants of a restaurant's strategy are: a) its capacity to attract Tourists $\left.\left(\lambda_{i}\right), \mathrm{b}\right)$ its quality $\left(x_{i, j}\right)$, and c) the expected equilibrium price $\left(\bar{p}_{T}^{*}\right)$; a) and b) can be considered as exogenous in the short run while c) is clearly endogenous. The dummy variables are meant to capture the heterogeneity in the arrival rates. In order to classify restaurants, we use the description in the guide. Restaurants classified as Restaurants for Tourists must satisfy at least one of the following conditions: a) restaurants for which the description explicitly states their customers are mainly tourists, b) restaurants located in particular tourist areas, c) restaurants with a particular view, d) hotel restaurants. Restaurants classified as restaurants for regulars are: a) restaurants for which the description explicitly states their customers are mainly regulars, b) restaurants for which the description uses the expression "out of tourist routes" or another equivalent expression, c) restaurants with some rare speciality. Restaurants which: a) fall in both previous classes, b) fall in neither of the previous classes, are classified as Popular Restaurants, a residual category. Thus, we consider as PR all restaurants which do not have a clear bias towards Tourists or Regulars. The procedure used to build RR, RT and PR can reasonably be assumed as exogenous in our empirical analysis. It relies on the exogenous capacity to attract Tourists rather than on restaurants' strategies.

We also consider the number of stars (which take the values 0,1,2,3 according to the quality of cuisine) as a proxy for quality. The dependent variable is the logarithmic price change for the interval 2002-03. ${ }^{3}$

Tab. 1 reports descriptive statistics for our sample. $\Delta P$ is the logarithmic difference of price. The first column shows a 3.6\% average increase of the price for the whole sample. The increase, however, has been considerably higher for those countries which adopted the euro (4.3\%) than for the others

\footnotetext{
${ }^{3}$ The guide reports a minimum and a maximum price for each restaurant. We only report the results for the minimum price, which is more informative for the purposes of our analysis. However, we have also performed tests for the maximum price with virtually identical outcomes. The results are available upon requests.
} 
(0.5\%). Individual country statistics are reported in Tab. 2. Italy, France and Germany are far above the average of non-euro countries. Standard deviations within euro countries are lower than within non-euro countries.

\subsection{Estimates}

We use OLS to estimate the following model:

$$
\begin{array}{r}
\Delta P_{i}=\beta_{0}+\beta_{1} E U R O R T_{i}+\beta_{2} E U R O R R_{i}+\beta_{3} E U R O P R_{i}+ \\
+\beta_{4} S T A R 1_{i}+\beta_{5} S T A R 2_{i}+\beta_{6} S T A R 3_{i}+u_{i}
\end{array}
$$

where $E U R O R T, E U R O R R$, and $E U R O P R$ are the products of the dummy $E U R O$ by $R T, R R$ and $P R$ respectively. The coefficients on these variables should be interpreted as the additional price changes due to the interaction of the euro effect with the restaurant's capacity to attract tourists. STAR1, $S T A R 2$, and $S T A R 3$ are dummies which take the value 1 according to the number of stars awarded to the individual restaurant by the guide. Including these variables allows to control for quality.

We use this model to test the key implications of our theoretical model. Proposition 3 suggests the rejection of the null hypothesis:

$$
H_{0}^{a}: \beta_{1}=\beta_{2}=\beta_{3}=0
$$

In fact, $\beta_{1}, \beta_{2}$, and $\beta_{3}$ can be interpreted as the differences in price change between euro and non-euro countries. The sum of the three variables exactly replicates a dummy for euro-countries. The significance of at least one parameter would indicate a different rate of inflation for euro-countries reative to non-euro-countries. The alternative hypothesis is thus that one or more parameters are jointly different from zero.

We also test Proposition 4. The null hypothesis in this case is:

$$
H_{0}^{b}: \beta_{1}=\beta_{2}
$$

It implies that the effect of the changeover is homogeneous among restaurants with different types of customers. By contrast, Proposition 4 suggests that in the presence of a jump the price change is higher for RT than for RR, while the price change for $\mathrm{PR}$ is ambiguous. Thus, the alternative hypothesis is that RT experienced higher price change than RR:

$$
H_{1}^{b}: \beta_{1}>\beta_{2}
$$

We also test the homogeneity between $P R$ on the one hand and $R R$ and $R T$ on the other. 
A consideration should be made. The guide selects restaurants on the basis of rigorous quality standards. This implies that our sample is biased toward quality restaurants. In other words, our sample does not include tourist traps. This suggests that we grossly underestimate the effect of the changeover.

Results are shown in Tab. 3. The coefficients of EURORR, EUROPR, are positive, but not significantly different from zero. The coefficient of EURORT is remarkably high and implies that tourist restaurants in the euro area have increased their prices by 6 percentage points more than non-euro restaurants. The coefficient for $P R\left(\beta_{3}\right)$ is between $\beta_{1}$ and $\beta_{2}$, as implied by the theoretical model. The intercept, that should be interpreted as the price change of non-euro restaurants (with no stars), is not significantly different from zero, implying that prices remained roughly constant outside the euro area.

The bottom part of the Table reports direct tests for our implications. $\mathcal{F}_{1}$ is an F-test for $H_{0}^{a}$. The null hypothesis of an homogeneous restaurant behaviour inside and outside the euro area is rejected at 0.1 percent confidence level. This finding strongly suggests the presence of a jump from a low to a high price equilibrium after the introduction of the euro.

$\mathcal{T}_{1}$ is a test for the null that the sum of $\beta_{1}, \beta_{2}$ and $\beta_{3}$ is equal to zero. The rejection of the null implies that the dummy EURO has a significant effect even without discriminating across different types of restaurants. $\mathcal{T}_{2}, \mathcal{T}_{3}$, and $\mathcal{T}_{4}$ are t-tests for the hypothesis of a homogeneous price change across restaurants with different customers: $\beta_{1}=\beta_{2}, \beta_{1}=\beta_{3}$, and $\beta_{2}=\beta_{3}$ respectively. $\mathcal{F}_{2}$ is an F-test for the joint hypothesis of homogeneity within restaurants with different customer-types: $\beta_{1}=\beta_{2}=\beta_{3}$.

The direct test for $H_{0}^{b}$ is thus the one-sided test $\mathcal{T}_{2}$. The main prediction of Proposition $4\left(\beta_{1}>\beta_{2}\right)$ is strongly supported by the data in both cases at $0.1 \%$ confidence level. The price change for tourist restaurants has been markedly higher than for restaurants with regular customers. The difference between $R T$ and $R R$ within euro-countries is about $4 \%$.

Additional insights can be obtained by considering $\mathcal{T}_{3}$ and $\mathcal{T}_{4}$. $\mathcal{T}_{3}$ rejects the null $\beta_{1}=\beta_{3}$ while $\mathcal{T}_{4}$ does not reject $\beta_{2}=\beta_{3}$. This evidence is consistent with the model prediction of an ambiguous behaviour of Popular Restaurants in the presence of a jump.

More surprising is the role of quality. The model predicts that low quality restaurants are on average more likely to deal only with tourists. By contrast, Tab. 3 shows that 2 and 3 stars restaurants have increased their prices significantly more than the others. However, this effect is not conditional on the introduction of the euro. A joint test for equality of coefficients inside and outside the euro area (not reported) could not reject the hypothesis at 
any common level of significance (the p-value is 0.486). Thus, the effect of top quality restaurants seems to be homogeneous among European countries regardless of the adoption of the new currency. A possible explanation is the occurrence of bad weather which caused an increase in food cost within Europe. Since high quality restaurants tend to consider different varieties of products as imperfect substitutes, they have a more limited choice of ingredients than other restaurants. Thus, in the event of an increase in food price, they are not able to choose those varieties which have been less affected by the weather. Hence, an increase in food cost might have affected top restaurants more than low quality restaurants.

An additional test of the validity of our model has been carried out in table 4, where results of a "difference-in-difference" regression are reported. The estimate of the euro-effect is positive and similar in size to table 3 . The average inflation differential between restaurants in the euro and non-euro areas is about $6.6 \%$ for $R T$ which is statistically greater than zero almost at $0.1 \%$ significance level. A direct test for the conditionality of the euro effect to the type of restaurants is $\mathcal{T}_{5}$ which tests whether the inflation differential between euro and non euro is the same for $R R$ and $R T$. The null is rejected at $1 \%$ significance level. ${ }^{4}$

The results shown are also robust to the introduction of the number of forks awarded to the individual restaurant. The number of forks (which takes values $1,2,3,4,5$ according to the venue's luxury and comfort) is a quality indicator more easily observable for customers than the quality of cuisine, and never results significant.

\section{Conclusions}

The introduction of the euro has had inflationary consequences on the service sector and in particular on the catering market. Restaurant prices have registered marked increases in all countries belonging to the euro zone. Far from being a neutral monetary reform, the adoption of a common currency has acted as a coordinating device, shifting the industry to a high-price equilibrium.

The theoretical predictions of our model of the catering market with multiplicity of equilibria are strongly supported by empirical evidence based on data from the Michelin Red Guide. The occurrence of abnormal price increases in countries belonging to the Euro area confirms the validity of our interpretation against popular alternative explanations such as increases in costs driven by bad weather.

\footnotetext{
${ }^{4}$ We are grateful to Antonio Ciccone for suggesting this test of robustness.
} 
The marked price increases in restaurants in tourist locations would seem to indicate the superiority of our theory also over the suggested justification in terms of rounding-off of prices in the new currency or overdue and delayed adjustment of menus.

The increase in the price of meals should not be regarded as a transitory blip destined eventually to revert back. Higher prices are here to stay unless an unlikely massive coordinated boycott by customers takes place.

Impressive as it is, the quantitative relevance of the phenomenon found in our empirical analysis is surely underestimated. Our results are, in fact, based on a sample of selected and reliable restaurants. The Michelin Red Guide is arguably one of the best and most exacting Restaurant guides in the world, where the truly bad tourist traps would never find place.

There has been a change in relative prices in Euroland, with possibly long-lasting redistributional effects in favour of the catering sector. 


\section{A Symmetric Equilibria}

Let us consider the possible cases:

1. $c\left(x_{L}\right)<p_{T}^{*} \leq v\left(x_{L}\right)<v\left(x_{M}\right)<v\left(x_{H}\right)$.

2. $c\left(x_{L}\right)<v\left(x_{L}\right)<p_{T}^{*}<v\left(x_{M}\right)<v\left(x_{H}\right)$.

3. $c\left(x_{L}\right)<v\left(x_{L}\right)<v\left(x_{M}\right) \leq p_{T}^{*}<v\left(x_{H}\right)$.

First case: $p_{T}^{*} \leq v\left(x_{L}\right)$

It is immediately possible to show that a Tourist will never be in the case $p_{T}^{*}<v\left(x_{L}\right)$. Since the lowest possible quality is $v\left(x_{L}\right)$, for $p_{T}^{*}<v\left(x_{L}\right)$ it always holds $p_{T}^{*}<E\left(v\left(x_{j}\right) \mid \bar{p}_{T}^{*}\right)$. In other words the Tourist is refusing too many profitable meals and would be better off with a higher reservation price. Thus, we can rule out this case. The case $p_{T}^{*}=v\left(x_{L}\right)$ is a bit more complex:

Lemma 2. The case $p_{T}^{*}=v\left(x_{L}\right)$ is a SNE if and only if $c\left(x_{M}\right) \geq v\left(x_{L}\right)$.

Proof. Assume $c\left(x_{M}\right) \geq v\left(x_{L}\right)$ and assume also that $v\left(x_{L}\right) \neq E\left(v\left(x_{j}\right) \mid v\left(x_{L}\right)\right)$ in presence of $p_{T}^{*}=v\left(x_{L}\right)$. This can happen only in the case that some medium or high quality restaurants are selling at $p_{T}^{*}$. But, if $c\left(x_{M}\right) \geq v\left(x_{L}\right)$ they are making non-positive profits and would be better off by dealing only with Locals. Thus, the equality $p_{T}^{*}=v\left(x_{L}\right)=E\left(v\left(x_{j}\right) \mid v\left(x_{L}\right)\right)$ must hold. Assume instead that $c\left(x_{M}\right)<v\left(x_{L}\right)$, then, by the expression for $\lambda_{M}^{P R}$ there is a non-zero fraction of medium quality restaurants that is dealing with tourists. Thus, it must be $E\left(v\left(x_{j}\right) \mid v\left(x_{L}\right)\right)>v\left(x_{L}\right)=p_{T}^{*}$.

Second case: $v\left(x_{L}\right)<p_{T}^{*}<v\left(x_{M}\right)$.

This is a more interesting case. In this case we have that $\lambda_{L}^{R T}-\underline{\lambda}$ low quality restaurants become PR (if they set $p=v\left(x_{L}\right)<p_{T}^{*}$ and $\bar{\lambda}-\lambda_{L}^{R T}$ become RT (by setting $p=p_{T}^{*}>v\left(x_{L}\right)$ ). Furthermore, $\lambda_{M}^{P R}-\underline{\lambda}$ medium quality restaurants become $\mathrm{RR}$ whereas $\bar{\lambda}-\lambda_{M}^{P R}$ become PR. The same applies for high quality restaurants. As for the restaurants, the Poisson nature of the process implies a discounted utility for a Tourist from a restaurant $i$ of quality $j$ equal to $\frac{\lambda_{i}}{\lambda_{i}+\delta} v\left(x_{j}\right)$. Thus, the expected quality given $\bar{p}_{T}^{*}$ is:

$$
\begin{aligned}
g\left(\bar{p}_{T}^{*}\right)=\theta\left(\int_{\underline{\lambda}}^{\bar{\lambda}} \frac{\lambda_{i}}{\lambda_{i}+\delta} v\left(x_{L}\right) d \lambda_{i}\right. & \left.+\int_{\lambda_{M}^{P R}}^{\bar{\lambda}} \frac{\lambda_{i}}{\lambda_{i}+\delta} v\left(x_{M}\right) d \lambda_{i}\right)+ \\
& +\theta\left(\int_{\lambda_{H}^{P R}}^{\bar{\lambda}} \frac{\lambda_{i}}{\lambda_{i}+\delta} v\left(x_{H}\right) d \lambda_{i}\right)
\end{aligned}
$$


where $\theta \equiv 1 /(\bar{\lambda}-\underline{\lambda})$. Note that the expression above depends on $\bar{p}_{T}^{*}$ through $\lambda_{M}^{P R}$ and $\lambda_{H}^{P R}$ which are just linear transformations of $\bar{p}_{T}^{*}$. Let denote them respectively:

$$
\lambda_{M}\left(\bar{p}_{T}^{*}\right) \equiv \lambda_{M}^{P R}=\frac{v\left(x_{M}\right)-\bar{p}_{T}^{*}}{v\left(x_{M}\right)-c\left(x_{M}\right)} \lambda
$$

and

$$
\lambda_{H}\left(\bar{p}_{T}^{*}\right) \equiv \lambda_{H}^{P R}=\frac{v\left(x_{H}\right)-\bar{p}_{T}^{*}}{v\left(x_{H}\right)-c\left(x_{H}\right)} \lambda
$$

Imposing symmetry $\left(p_{T}^{*}=\bar{p}_{T}^{*}\right)$ and the equilibrium condition $g\left(\bar{p}_{T}^{*}\right)=p_{T}^{*}$ we obtain:

$$
\begin{aligned}
& p_{T}^{*}=\theta a_{0} v\left(x_{L}\right)+\theta a_{1}\left(v\left(x_{M}\right)+v\left(x_{H}\right)\right)+ \\
& +\theta v\left(x_{M}\right)\left(\delta \ln \left(\lambda_{M}\left(p_{T}^{*}\right)+\delta\right)-\lambda_{M}\left(p_{T}^{*}\right)\right)+ \\
& \quad+\theta v\left(x_{H}\right)\left(\delta \ln \left(\lambda_{H}\left(p_{T}^{*}\right)+\delta\right)-\lambda_{H}\left(p_{T}^{*}\right)\right)
\end{aligned}
$$

where:

$$
a_{0} \equiv \bar{\lambda}-\underline{\lambda}-\delta \ln (\bar{\lambda}+\delta)+\delta \ln (\underline{\lambda}+\delta)
$$

and

$$
a_{1} \equiv \bar{\lambda}-\delta \ln (\bar{\lambda}+\delta)
$$

and the right hand side in (A.2) is clearly nonlinear in $p_{T}^{*}$.

Third Case: $v\left(x_{M}\right) \leq p_{T}^{*}<v\left(x_{H}\right)$ In general, $v\left(x_{M}\right)=p_{T}^{*}$ is not an equilibrium unless for particular values of the parameters. Consider what happens if $p_{T}^{*}>v\left(x_{M}\right)$. In this case, again $\lambda_{L}^{R T}-\underline{\lambda}$ low quality restaurants become $\mathrm{PR}$ and $\bar{\lambda}-\lambda_{L}^{R T}$ become RT. Moreover, $\lambda_{M}^{R T}-\underline{\lambda}$ medium quality restaurants become $\mathrm{PR}$ and $\bar{\lambda}-\lambda_{M}^{R T}$ become RT (thus, if the price is high enough, also medium quality restaurants become RT). Finally, $\lambda_{H}^{P R}-\underline{\lambda}$ become RR and $\bar{\lambda}-\lambda_{H}^{P R}$ become $\mathrm{PR}^{5}$. The expected quality for a Tourist is:

$$
\begin{array}{r}
g\left(\bar{p}_{T}^{*}\right)=\theta\left(\int_{\underline{\lambda}}^{\bar{\lambda}} \frac{\lambda_{i}}{\lambda_{i}+\delta} v\left(x_{L}\right) d \lambda_{i}\right. \\
\left.+\int_{\underline{\lambda}}^{\bar{\lambda}} \frac{\lambda_{i}}{\lambda_{i}+\delta} v\left(x_{M}\right) d \lambda_{i}\right)+ \\
+\theta\left(\int_{\lambda_{H}^{P R}}^{\bar{\lambda}} \frac{\lambda_{i}}{\lambda_{i}+\delta} v\left(x_{H}\right) d \lambda_{i}\right)
\end{array}
$$

and, using the same notation as in (A.2), the equilibrium is:

$$
\begin{aligned}
& p_{T}^{*}=\theta a_{0}\left(v\left(x_{M}\right)+v\left(x_{L}\right)\right)+\theta a_{1} v\left(x_{H}\right)+ \\
& \quad+\theta v\left(x_{H}\right)\left(\delta \ln \left(\lambda_{H}\left(p_{T}^{*}\right)+\delta\right)-\lambda_{H}\left(p_{T}^{*}\right)\right)
\end{aligned}
$$

\footnotetext{
${ }^{5}$ Note that the cutoffs $\lambda_{L}^{R T}$ and $\lambda_{H}^{P R}$ are different from the previous case since $p_{T}^{*}$ is now higher.
} 
and, again, the expected quality is nonlinear in the price. When the price $\bar{p}_{T}^{*}$ which is expected to prevail is lower than $v\left(x_{M}\right)$ the relevant function is that in (A.2). When $\bar{p}_{T}^{*} \geq v\left(x_{M}\right)$ the relationship (A.3) holds. 


\section{References}

European Central Bank. Monthly Bulletin. ECB, April 2002.

European Central Bank. Monthly Bulletin. ECB, July 2002.

European Central Bank. Monthly Bulletin. ECB, March 2002.

Y. Chan and H. Leland. Prices and qualities in markets with costly information. Review of Economic Studies, 49(4):499-516, 1982.

R. Cooper and T. W. Ross. Prices, product qualities and asymmetric information: The competitve case. Review of Economic Studies, 51(2):197-207, 1984.

Michelin Guide. The Red Guide: Main Cities of Europe. Michelin Travel Publications, France, 2002 and 2003 editions.

P. Nelson. Information and consumer behavior. Journal of Political Economy, 78(2):311-329, 1970.

S. Salop and J. Stiglitz. A model of monopolistically competitive price dispersion. Review of Economic Studies, 44(3):493-510, 1977.

T. von Ungern-Sternberg and C. C. von Weizsacker. The supply of quality on a market for "experience goods". Journal of Industrial Economics, 33 (4):531-540, 1985. 
Table 1: Descriptive Statistics

\begin{tabular}{cccc}
\hline & All Sample & Euro & Non Euro \\
& $\Delta P$ & $\Delta P$ & $\Delta P$ \\
\hline Mean & 0.033 & 0.043 & 0.005 \\
S.d. & 0.161 & 0.129 & 0.227 \\
Min & -1.374 & -0.624 & -1.374 \\
Max & 0.951 & 0.951 & 0.681 \\
Obs. & 746 & 552 & 194 \\
\hline
\end{tabular}

Notes: $\Delta P$ is the logarithmic difference of the price between the 2003 and the 2002 editions. 
Table 2: Country Averages

\begin{tabular}{ccc}
\hline Country & $\Delta P$ & Obs. \\
& $($ S.d. $)$ & \\
\hline Denmark & -0.001 & 11 \\
& $(0.272)$ & \\
Sweden & -0.014 & 28 \\
& $(0.269)$ & \\
UK & 0.009 & 155 \\
& $(0.217)$ & \\
Italy & 0.055 & 155 \\
& $(0.134)$ & \\
France & 0.051 & 216 \\
& $(0.135)$ & \\
Germany & 0.025 & 181 \\
& $(0.115)$ & \\
\hline
\end{tabular}


Table 3: OLS estimates for Equation (8)

\begin{tabular}{cc}
\hline & Dependent Var. \\
Variable & $\Delta P$ \\
\hline EURORT & $0.060^{*}$ \\
& $(0.019)$ \\
EURORR & 0.017 \\
& $(0.018)$ \\
EUROPR & 0.021 \\
& $(0.021)$ \\
STAR1 & 0.024 \\
& $(0.014)$ \\
STAR2 & $0.048^{*}$ \\
& $(0.015)$ \\
STAR3 & $0.044^{*}$ \\
& $(0.017)$ \\
cons & 0.001 \\
& $(0.017)$ \\
$R^{2}$ & 0.035 \\
\hline $\mathcal{F}_{1}: F_{(3,739)}$ & $6.06^{* * *}$ \\
p-value & $(0.000)$ \\
$\mathcal{F}_{2}: F_{(2,739)}$ & $7.17^{* * *}$ \\
p-value & $(0.001)$ \\
$\mathcal{T}_{1}: t_{739}$ & $1.90^{*}$ \\
p-value & $(0.029)$ \\
$\mathcal{T}_{2}: t_{739}$ & $3.73^{* * *}$ \\
p-value & $(0.000)$ \\
$\mathcal{T}_{3}: t_{739}$ & $2.38^{*}$ \\
p-value & $(0.018)$ \\
$\mathcal{T}_{4}: t_{739}$ & 0.28 \\
p-value & $(0.784)$ \\
\hline &
\end{tabular}

Notes: The table reports OLS estimates for Equation (8) with heteroscedasticity robust standard errors (in parentheses). Number of observations $=746$. Tests for the following hypotheses are reported:

$\mathcal{F}_{1}: E U R O R T=E U R O P R=E U R O R R=0$.

$\mathcal{F}_{2}: E U R O R T=E U R O P R=E U R O R R$.

$\mathcal{T}_{1}:$ EURORT + EURORR + EUROPR $=0$ against EURORT + EURORR + $E U R O P R>0$ (one-sided).

$\mathcal{T}_{2}: E U R O R T=E U R O R R$ against $E U R O R T>E U R O R R$ (one-sided).

$\mathcal{T}_{3}: E U R O R T=E U R O P R$ (two-sided).

$\mathcal{T}_{4}: E U R O P R=E U R O R R$ (two-sided).

${ }^{*}=5 \%$ significant. ${ }^{* *}=1 \%$ significant. ${ }^{* * *}=0.1 \%$ significant. 
Table 4: Difference-in-Difference Estimation

\begin{tabular}{cc}
\hline & Dependent Var. \\
Variable & $E U R O \Delta P-(1-E U R O) \Delta P$ \\
\hline RT & $0.060^{* * *}$ \\
& $(0.017)$ \\
RR & 0.020 \\
& $(0.016)$ \\
cons & 0.006 \\
& $(0.014)$ \\
$R^{2}$ & 0.021 \\
\hline $\mathcal{T}_{5}: t_{743}$ & $3.07^{* *}$ \\
p-value & $(0.001)$ \\
$\mathcal{T}_{6}: t_{743}$ & $6.27^{* * *}$ \\
p-value & $(0.000)$ \\
$\mathcal{T}_{7}: t_{743}$ & $3.20^{* *}$ \\
p-value & $(0.002)$ \\
\hline
\end{tabular}

Notes: The table reports OLS estimates for the following model:

$$
y=\gamma_{0}+\gamma_{1} R T+\gamma_{2} R R+\epsilon
$$

where $y \equiv E U R O \Delta P-(1-E U R O) \Delta P$. Heteroscedasticity robust standard errors are in parentheses. Number of observations $=746$. Tests for the following hypotheses are reported:

$\mathcal{T}_{5}: R T=R R$ against $R T>R R$ (one-sided).

$\mathcal{T}_{6}: R T+$ cons $=0$.

$\mathcal{T}_{7}: R R+$ cons $=0$.

$*=5 \%$ significant. ${ }^{*} *=1 \%$ significant. ${ }^{* * *}=0.1 \%$ significant. 
Figure 1: Multiple Equilibria

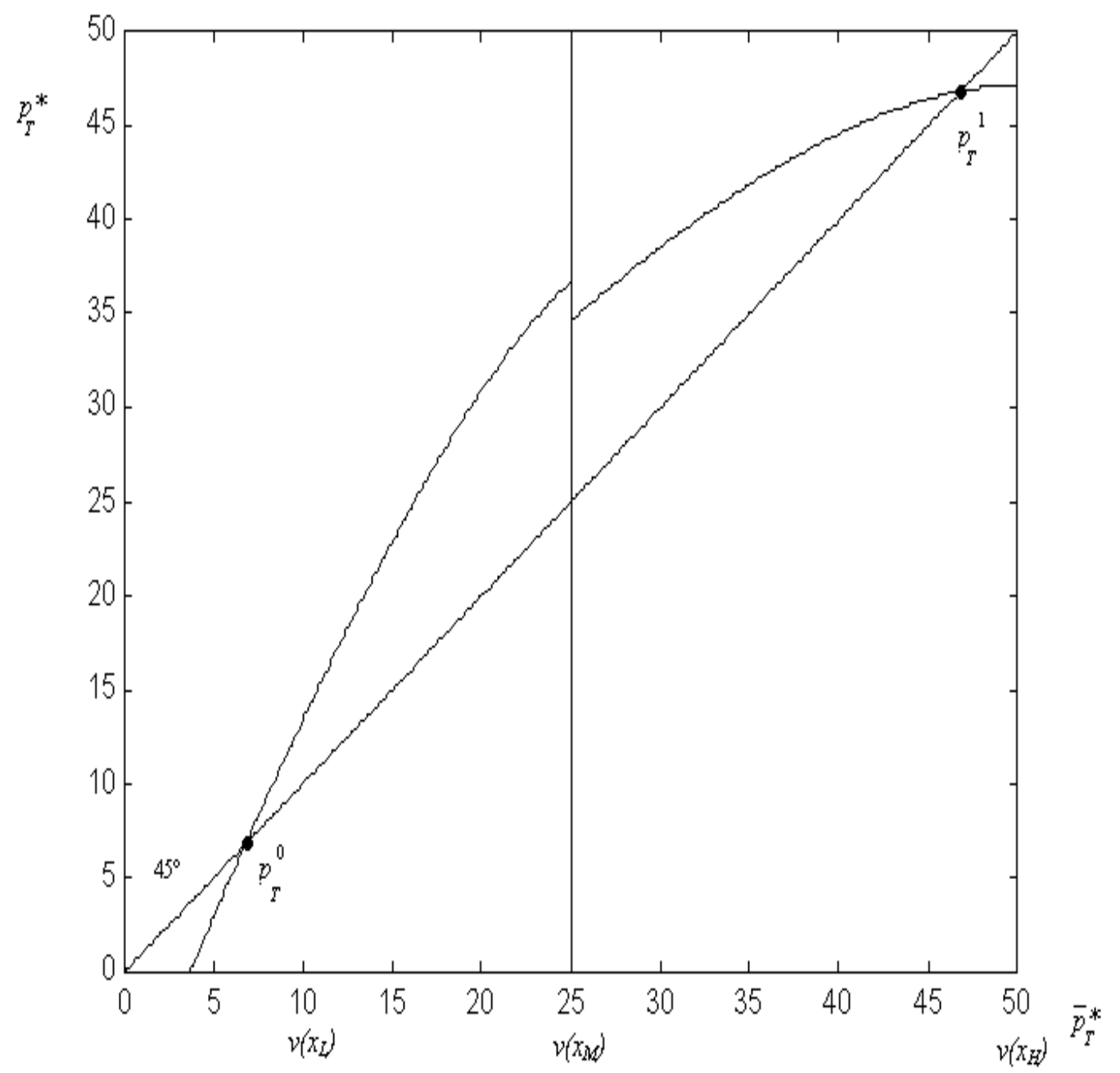

The figure shows the possibility of multiple equilibria. It is based on the following set of parameters:

\begin{tabular}{cccccc}
\hline$v\left(x_{L}\right)$ & 10 & $c\left(x_{L}\right)$ & 1 & $\bar{\lambda}$ & 1 \\
$v\left(x_{M}\right)$ & 25 & $c\left(x_{M}\right)$ & 5 & $\underline{\lambda}$ & 0.3 \\
$v\left(x_{H}\right)$ & 50 & $c\left(x_{H}\right)$ & 7 & $\lambda$ & 1 \\
$\delta$ & .8 & & & & \\
\hline
\end{tabular}

